\title{
CubeSat laser infrared crosslinK mission status
}

Paul Serra, Hannah Tomio, Ondrej Cierny, William Kammerer, Peter Grenfell, et al.

Paul C. Serra, Hannah Tomio, Ondrej Cierny, William Kammerer, Peter Grenfell, Grant Gunnison, Joseph Kusters, Cadence Payne, Paula do Vale Pereira, David Mayer, Jan Stupl, John Hanson, Simon Barke, Myles Clark, Tyler Ritz, Danielle Coogan, John Conklin, Kerri L. Cahoy, "CubeSat laser infrared crosslinK mission status," Proc. SPIE 11852, International Conference on Space Optics - ICSO 2020, 118523D (11 June 2021); doi: 10.1117/12.2599543

SPIE Event: International Conference on Space Optics - ICSO 2021, 2021, Online Only 


\section{International Conference on Space Optics-ICSO 2020}

Virtual Conference

30 March-2 April 2021

Edited by Bruno Cugny, Zoran Sodnik, and Nikos Karafolas
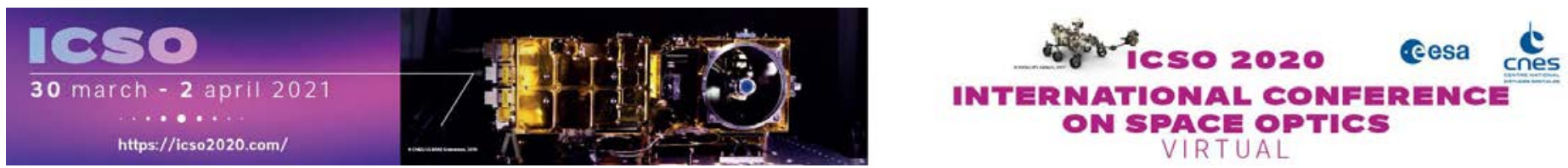

\section{CubeSat laser infrared crosslinK mission status}

\section{Cesa isopmeatians ecnes}




\title{
CubeSat Laser Infrared CrosslinK Mission Status
}

\author{
Hannah Tomio ${ }^{a}$, Paul Serra ${ }^{a}$, Ondrej Čierny ${ }^{a}$, William Kammerer ${ }^{\mathrm{a}}$, Peter Grenfell ${ }^{\mathrm{a}}$, Grant \\ Gunnison $^{\mathrm{a}}$, Joseph Kusters ${ }^{\mathrm{a}}$, Cadence Payne ${ }^{\mathrm{a}}$, Paula Do Vale Pereira ${ }^{\mathrm{a}}$, David Mayer ${ }^{\mathrm{b}}$, Jan \\ Stupl $^{\mathrm{b}}$, John Hanson ${ }^{\mathrm{c}}$, Simon Barke ${ }^{\mathrm{d}}$, Myles Clark ${ }^{\mathrm{d}}$, Tyler Ritz ${ }^{\mathrm{d}}$, Danielle Coogan ${ }^{\mathrm{d}}$, John \\ Conklin $^{\mathrm{d}}$, and Kerri Cahoy ${ }^{\mathrm{a}}$ \\ ${ }^{a}$ Massachusetts Institute of Technology, Department of Aeronautics and Astronautics, 77 \\ Mass. Ave., Cambridge, MA 02139, USA \\ bNASA Ames Research Center, Moffett Blvd., Mountain View, CA 94035, USA \\ ${ }^{\mathrm{c}}$ CrossTrac Engineering, 2730 Saint Giles Ln., Mountain View, CA 94040-4437, USA \\ ${ }^{\mathrm{d} U n i v e r s i t y ~ o f ~ F l o r i d a, ~ D e p a r t m e n t ~ o f ~ M e c h a n i c a l ~ \& ~ A e r o s p a c e ~ E n g i n e e r i n g, ~} 231$ MAE-A, P.O. \\ Box 116250, Gainesville, FL 32611, USA
}

\begin{abstract}
Constellations of CubeSats will benefit from high data rate communications links and precision time transfer and ranging. The CubeSat Laser Infrared CrosslinK (CLICK) mission intends to demonstrate low size, weight, and power (SWaP) laser communication terminals, capable of conducting full-duplex high data rate downlinks and crosslinks, as well as high precision ranging and time transfer. A joint project between the Massachusetts Institute of Technology (MIT), the University of Florida (UF), and NASA Ames Research Center, CLICK consists of two separate demonstration flights: the initial CLICK-A, which will demonstrate a space-to-ground downlink and serve as a risk-reduction mission, and CLICK-B/C, a crosslink demonstration mission.

The CLICK payloads each consist of laser transceivers and pointing, acquisition, and tracking (PAT) systems, and will fly on $3 \mathrm{U}$ CubeSat buses from Blue Canyon Technologies to perform their optical downlink and crosslink experiments in low Earth orbit (LEO). We present an update on the status of both the CLICK-A and CLICK-B/C payloads. At the time of writing, the final assembly and testing of the CLICK-A payload has been completed and the payload has been delivered for integration with the spacecraft bus. The final testing included the validation of the transmitter and the PAT system, the performance of both of which was characterized under various environmental test conditions and shown to meet their requirements for operation on orbit. On CLICK-B/C, the payload electronics have been designed and are under test. The optical bench of the payload has been assembled, the characterization of which is ongoing.
\end{abstract}

Keywords: laser, optical, crosslink, intersatellite, CubeSat, communications

\section{INTRODUCTION}

The past decade has brought a paradigm shift to space missions and systems, evidenced by the growing numbers of CubeSats and other nanosatellites in orbit. The increasing availability of low-cost launch services and commercial off-the-shelf (COTS) components, coupled with new design approaches that borrow from iterative development and rapid prototyping practices, have greatly lowered the barriers to entry to space and fostered significant technological advancements. Time and again, these small satellites have proven to be highly capable platforms for a wide variety of scientific instruments and commercial applications. They offer particular advantages when launched and operated in concert to form constellations of large numbers of spacecraft, enabling improved spatial and temporal coverage of the Earth. Recent years have seen advanced mission concepts, such as those involving instruments capable of hyperspectral or high-resolution imaging, or coordinated operations for interferometry or bistatic sensing configurations. However, such concepts have challenging requirements for connectivity, including high data rate communication and precision ranging and time transfer. Meeting these

Further author information: (Send correspondence to H.T.)

H.T.: E-mail: tomio@mit.edu 
requirements with traditional radio frequency (RF) systems is difficult due to the size, weight, and power (SWaP) constraints of small satellites. Furthermore, the RF spectrum is heavily regulated due to finite bandwidth, and the cost and complexity of the licensing process for certain frequency allocations can be prohibitive.

Laser communications (lasercom) technologies have the potential to avoid these disadvantages of RF systems and even exceed their capabilities. Their smaller wavelength implies a narrower beamwidth and higher directionality, allowing lasercom systems to be more SWaP efficient. In addition, the optical spectrum is subject to few licensing constraints and the narrow beamwidth lessens the risk of interception, providing greater security for a communication link. For ranging and timing in particular, the use of optical frequencies can enable orders of magnitude improvements over RF systems.

\subsection{CLICK Mission}

The CubeSat Laser Infrared CrosslinK (CLICK) mission will demonstrate low SWaP laser communication terminals, capable of conducting full-duplex high data rate downlinks and crosslinks, as well as high precision ranging and time transfer. A joint project between the Massachusetts Institute of Technology (MIT), the University of Florida (UF), and NASA Ames Research Center, CLICK consists of two separate demonstration flights: the initial CLICK-A, which will demonstrate a space-to-ground downlink and serve as a risk-reduction mission, and CLICK-B/C, a crosslink demonstration mission. The CLICK technology demonstration will ultimately enable mission concepts including space radio interferometry, GPS-denied navigation, time synchronization for synthetic aperture telescopes, and improved bandwidth for science data telemetry on SWAP-limited platforms like nanosatellites.

The CLICK mission has its origins in MIT's Nanosatellite Optical Downlink Experiment (NODE), previously described in Refs. 1 and 2, and leverages the design of UF's Miniature Optical Communications Transmitter (MOCT). ${ }^{3,4}$ The CLICK payloads, consisting of laser transceivers and pointing, acquisition, and tracking (PAT) systems, will each fly on a $3 \mathrm{U}$ CubeSat bus from Blue Canyon Technologies. The optical downlink and crosslink experiments will be performed in low Earth orbit (LEO), at an altitude of roughly $400 \mathrm{~km}-600 \mathrm{~km}$. In a concurrent effort, a portable optical ground station (OGS) is being developed for the CLICK mission. The $30 \mathrm{~cm}$ aperture Portable Telescope for Lasercom (PorTeL), which features a beacon laser uplink, receiver system, and coarse and fine pointing capabilities, will serve as the ground receiver for the downlink demonstration. ${ }^{5}$

This paper provides an overview of the CLICK-A flight demonstration by describing the mission concept of operations (ConOps) and payload specifications. In addition, the current status of the mission is described. At the time of writing, the final assembly and testing of the CLICK-A payload has been completed and the payload has been delivered for integration with the spacecraft bus. This paper covers the final stages of assembly, integration, and testing for CLICK-A and discusses the procedures and results of the tests, including the functional testing of the transmitter and the pointing, acquisition, and tracking (PAT) system. We discuss implications of these results for on-orbit operations. Finally, the CLICK-BC flight demonstration is described and an update on its current status is presented.

\section{CLICK-A}

The primary objective of the CLICK-A mission is to demonstrate an optical downlink with a data rate of $\geq 10 \mathrm{Mbps}$ to the PorTeL OGS. This demonstration will validate the design of the payload's laser transmitter and fine pointing stage, as well as that of the optical ground station.

\subsection{CLICK-A ConOps}

The ConOps of the demonstration are shown in Fig. 1. Following the initial deployment from the International Space Station (ISS), RF communication will be established with the spacecraft using the KSATLite ground station network. KSATLite ground stations will transmit commands to and receive telemetry and mission data from the spacecraft, and the network will relay this information to the mission operations center (MOC), which is located at MIT. The MOC will conduct passes with the KSATLite ground stations and coordinate all spacecraft operations, beginning with a functional check-out of the payload subsystem and including the optical downlink experiments. 


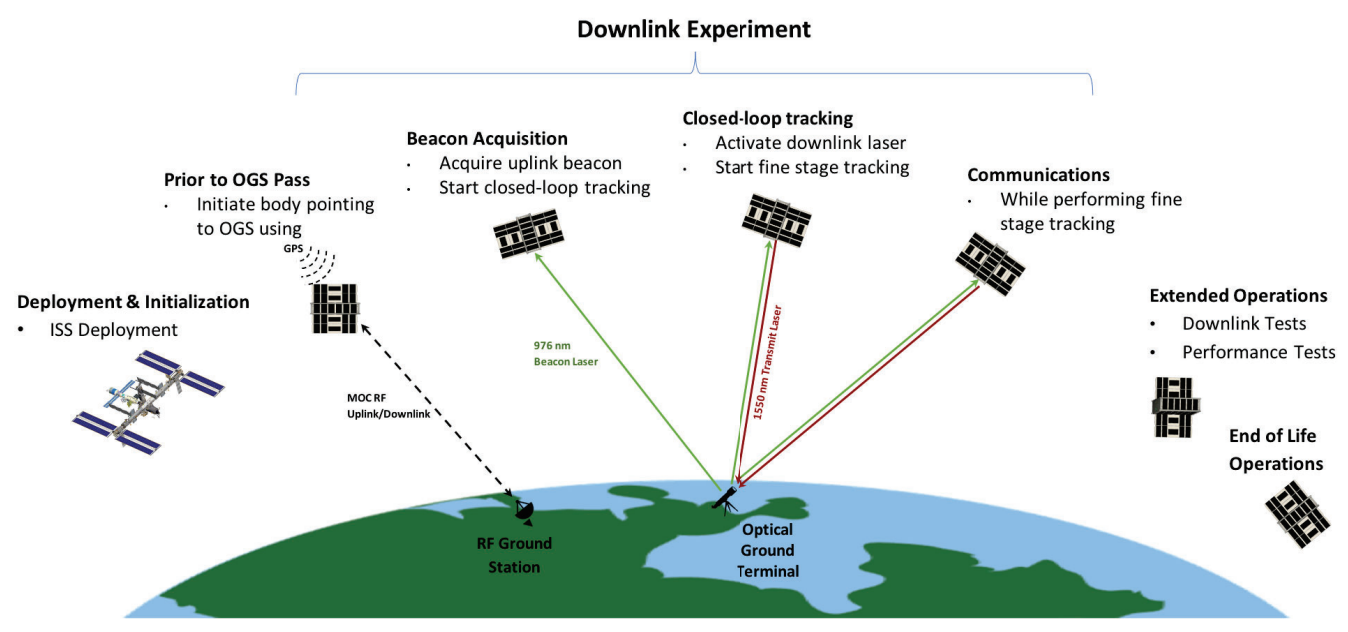

Figure 1: CLICK-A Mission Concept of Operations

The optical downlink experiment is conducted in several stages. Prior to the start of a pass, the spacecraft bus initiates its attitude control sequence to point the payload aperture at the optical ground station. The bus system also powers on the payload to enable heating and initial boot-up. At the beginning of the pass itself, the uplink beacon laser is transmitted from the OGS. Specifications of this laser and the payload transmitter are given in Table 1. On ground, the OGS operates in coarse stage tracking in open loop (CSTOL) mode, in which it will track the spacecraft based on ephemeris data generated by the on-board GPS receiver. The ephemeris data is obtained prior to the start of the demonstration, having been downlinked during a previous RF pass with a KSATLite ground station.

Table 1: CLICK-A Laser Specifications

\begin{tabular}{|l|c|c|c|c|}
\hline & Wavelength & Average Power & Beam Divergence & Modulation \\
\hline Beacon Laser & $976 \mathrm{~nm}$ & $2.5 \mathrm{~W}$ & $0.32^{\circ}$ FWHM & Sine wave \\
\hline Transmitter Laser & $1550 \mathrm{~nm}$ & $200 \mathrm{~mW}$ & $1.3 \mathrm{mrad}$ FWHM & M-ary PPM \\
\hline
\end{tabular}

Using the body pointing of the spacecraft, the beacon laser signal is detected and acquired using the beacon tracking camera. This initiates the closed-loop fine stage tracking (FST) mode, which uses the payload's fine steering mirror (FSM). The FSM is controlled using the error offset on the camera sensor between the beacon spot and a spot generated by an on-board calibration laser that has been multiplexed with the payload transmit laser. For more detail, see Ref. 6 . With the payload conducting fine pointing, the payload downlink laser is detected by the OGS using its own near-infrared (NIR) tracking camera. The OGS will subsequently operate for the duration of the pass in its coarse stage tracking in closed loop (CSCTL) mode, in which it will track the downlink laser from the spacecraft instead of relying on the ephemeris data. In the final stage of the experiment, the OGS will enter FST mode as well, using its own internal FSM which is similarly controlled using the tracking camera error signal. In this mode, the downlink laser from the spacecraft will be centered on the receiver avalanche photodiode (APD). The signal from the APD is sampled using an oscilloscope throughout the pass for offline analysis. $^{7}$

After the pass has ended, the payload will perform any necessary shut-down activities, such as data logging and transferring, before being switched off by the spacecraft bus.

\subsection{CLICK-A Payload Design}

The CLICK-A payload consists of a laser transmitter and fine pointing PAT system in a $1.2 \mathrm{U}$ form-factor. As shown in Fig. 2, the maximum envelope of the payload is roughly $96 \mathrm{~mm} \times 96 \mathrm{~mm} \times 138 \mathrm{~mm}$ in size, and it has 


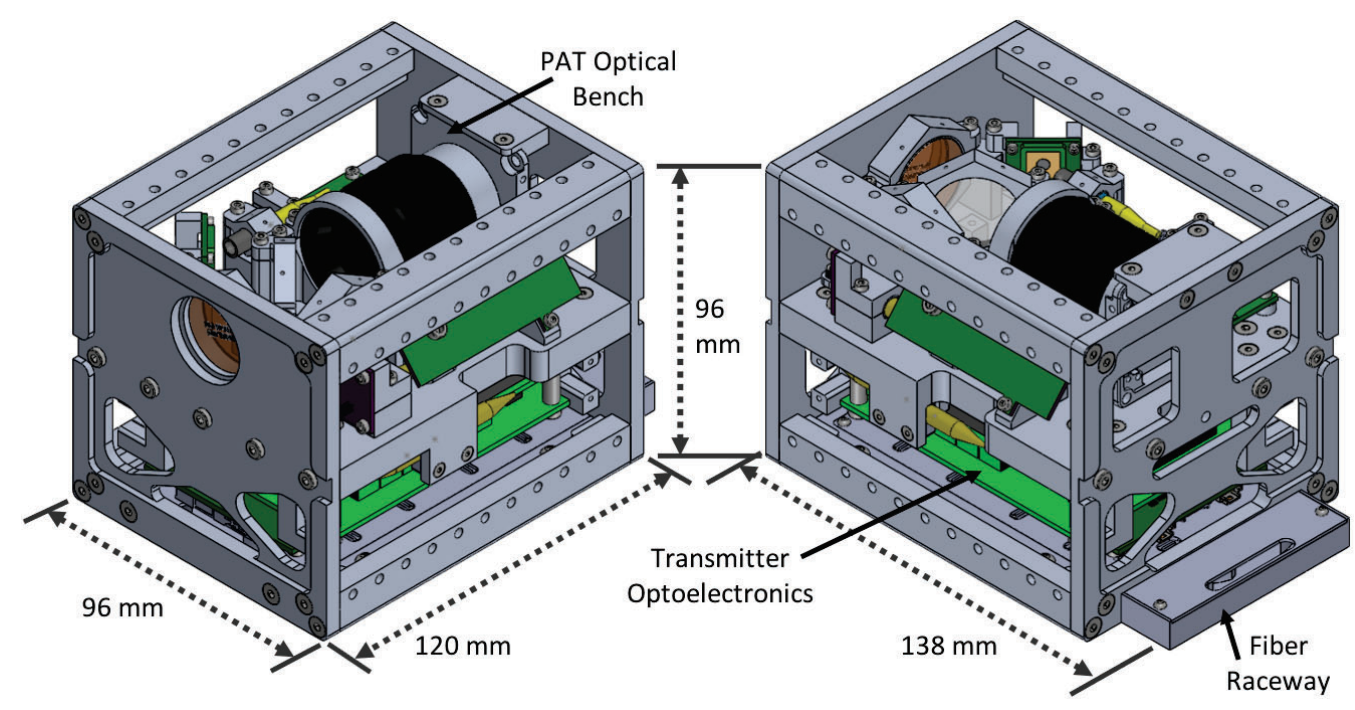

Figure 2: CLICK-A Payload Structure

a mass of approximately $1.17 \mathrm{~kg}$. The upper half of the payload hosts the optical bench for the fine pointing system, while the lower half of the payload contains the transmitter optoelectronics. Both of these halves sit above the fiber raceway. The mechanical assembly is further detailed in Fig. 3. The optical bench includes the beacon tracking camera, a Matrix Vision mvBlueFOX, and its lens assembly, a Schneider Xenoplan lens, as well as the FSM (from Mirrorcle Technologies) used to conduct beam steering. The payload optomechanical design, which supports the ConOps described in the Section 2.1, is summarized in Fig. 4.

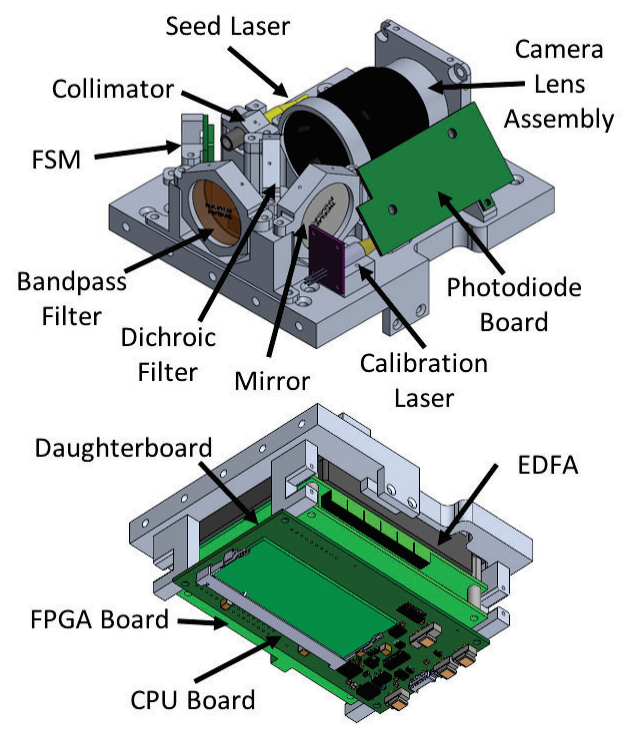

Figure 3: CLICK-A Payload Mechanical Design

The $1550 \mathrm{~nm}$ transmit laser and $980 \mathrm{~nm}$ calibration laser, coupled through wavelength division multiplexing (WDM), are collimated and directed to the FSM. The FSM conducts fine pointing by steering the beam to the dichroic beam splitter (DBS). The transmit laser is reflected and exits via the payload aperture, while the calibration laser is directed using a mirror to the lens assembly and the camera's focal plane array. The $976 \mathrm{~nm}$ 
uplink beacon signal follows a similar path through the DBS to the camera and lens assembly. A double bandpass filter is used at the aperture for stray light isolation.

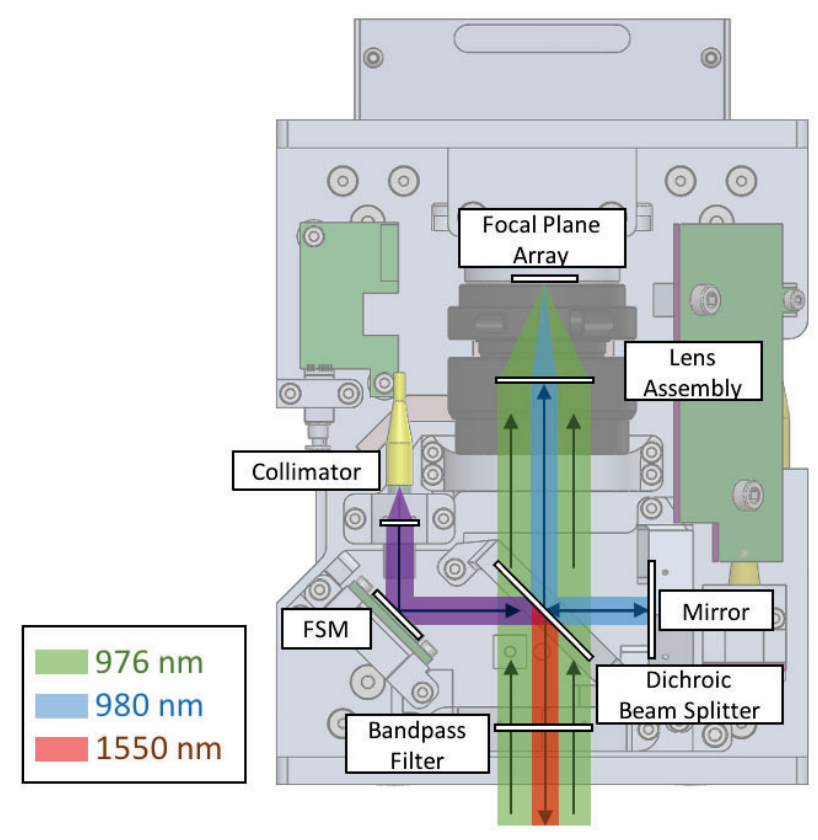

Figure 4: CLICK-A Payload Optical Design

The lower half of the payload, as illustrated in Fig. 3, contains the transmitter optoelectronics, including a COTS Erbium-Doped Fiber Amplifier (EDFA) and modulation and control electronics board stack. The primary controller of the payload is the Raspberry Pi-based CPU board, which provides command and data handling and interfaces with the spacecraft bus. An additional microcontroller is utilized to enable the Raspberry $\mathrm{Pi}$ to be reprogrammed on orbit. The CPU board also interfaces with the beacon camera and runs the software for the PAT sequence. A field programmable gate array (FPGA) is utilized for the control and modulation of the transmitter seed laser. The transmitted signal is modulated using M-ary pulse position modulation (PPM), which is implemented with a pulse duration of $5 \mathrm{~ns}$ and a variable PPM order ranging from 4 to 128. The FPGA, a Xilinx Spartan 6, is located on its own board with additional circuitry for current sensing and interfacing with the daughterboard. The daughterboard is primarily an interface board for the signals from the FPGA to the various other components. It includes interfaces and driver electronics for the payload heaters, resistance temperature detectors (RTDs), FSM, photodiodes, calibration laser, and transmitter seed laser. This system architecture is shown in Fig. 5.

The $1550 \mathrm{~nm}$ downlink transmitter uses a master-oscillator power-amplifier (MOPA) design based on a COTS transmitter optical sub-assembly (TOSA) for the seed laser and an EDFA for the optical amplifier. The transmitter design is based on that of NODE, as previously described in Refs. 8-1. The FPGA controls the bias current and temperature (using a thermo-electric cooler, or TEC) of the seed laser diode in order to shift the output signal wavelength in and out of the passband of a Fiber Bragg Grating (FBG), thereby achieving a high extinction ratio. A modulated signal from the FPGA, along with the TEC control, is applied to the TOSA to induce a signal of a certain wavelength which is then directed via optical fiber to the FBG through a circulator. Wavelengths within the passband of the FBG, signifying a PPM pulse, are reflected back into the circulator and directed to the EDFA. Wavelengths outside the passband, indicating an empty PPM time slot, are transmitted through the FBG to an attenuator and photodetector. The EDFA amplifies the signal and directs it to a collimator to achieve the specified beam divergence. This design is illustrated in Fig. 6.

Three PIN photodiodes are used to measure the optical power at various points to provide feedback and allow 


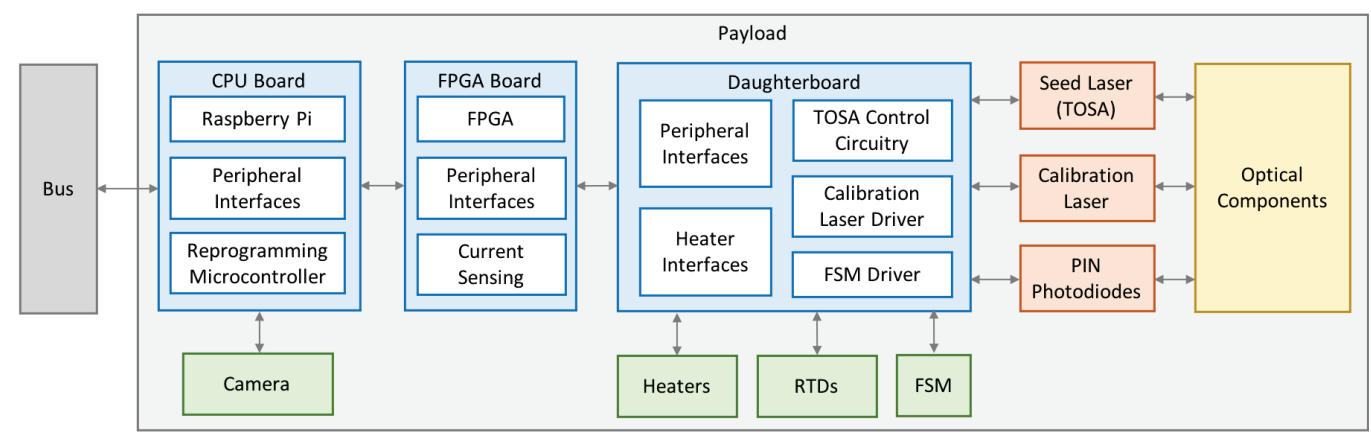

Figure 5: CLICK-A Payload System Architecture

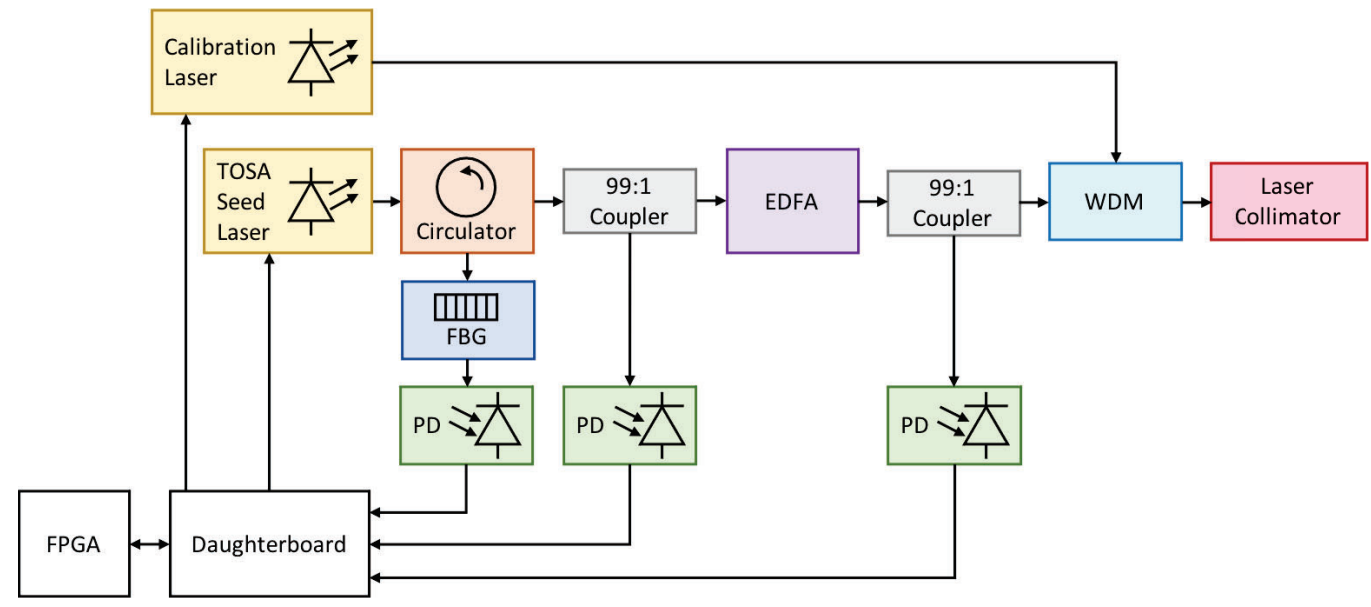

Figure 6: CLICK-A Transmitter Architecture

closed-loop control of the transmitter. The FPGA utilizes these measured values to adjust the bias current and temperature of the laser diode to finely tune the transmitter wavelength. This also enables the built-in self-test (BIST) capability of the transmitter to verify the output signal.

A comprehensive discussion of the transmitter design can be found in Ref. 8. The performance of this system has previously been evaluated and an analysis of the communication link has been completed in Refs. 6-7.

\subsection{CLICK-A Status}

Recently, the final assembly and testing of the CLICK-A payload has been completed and the payload has been delivered for integration with the spacecraft bus. The fully assembled flight model is shown in Fig. 7. The final testing of the payload focused on the validation of the optoelectronic and mechanical design of the transmitter and PAT system. In parallel, the payload flight software was developed, tested, and finalized as well. For environmental tests, the assembled payload was subject to thermal vacuum testing in various thermal conditions. Ultimately, the testing campaign conducted at MIT sought to demonstrate the functional operation of the payload and characterize its performance. Select results from this testing are presented and discussed here.

To enable the functional testing of the payload on ground, an all-in-one instrument for the ground support equipment (GSE) was developed. The design of the GSE is illustrated in Fig. 8. Equipped with its own beacon laser, FSM, APD, and IR camera, as well as an $M^{2}$ measurement system from Thorlabs for beam profiling, the GSE enables the measurement and characterization of the transmitter beam quality and divergence, received signal pulse shape and amplitude, and the accuracy of the PAT system. In order to streamline testing further, 


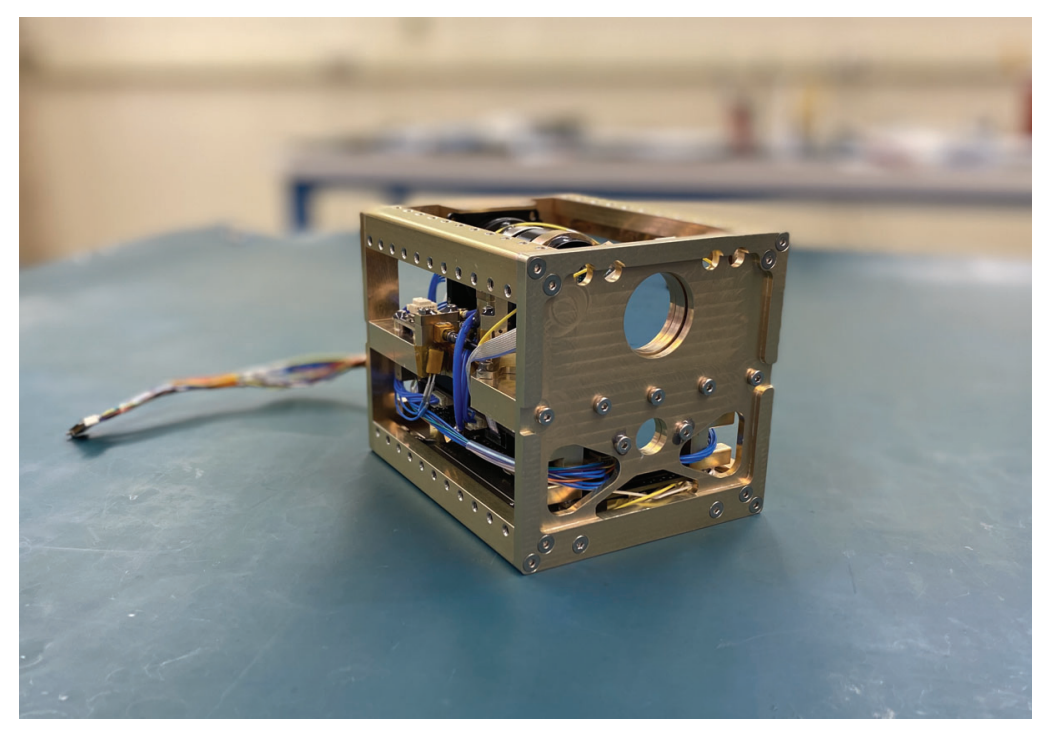

Figure 7: CLICK-A Payload Flight Model

automated scripts were developed to run with the payload controller and GSE. These self-test scripts will also be run via command on orbit to check the functionality of the payload.

The testing of the transmitter included confirming the seed laser power and modulation, characterizing the pulse shape, measuring the peak power of the EDFA, and examining the beam quality in a variety of environmental conditions. Figure 9 shows the power of the seed laser for the CLICK-A payload over its output wavelength ranges at various PPM orders as well as the insertion loss of the FBG used for modulation. Due to chirp, the wavelength of the seed laser is shifted during pulses. The amplitude at the shifted wavelength, around $1550.13 \mathrm{~nm}$, is proportional to the duty cycle and is divided by 2 for each increase in PPM order. The light sent to the EDFA is the amount reflected on the FBG, so the convolution of the insertion loss and the seed laser power.

In addition, the transmitter peak output power was recorded at various duty cycles during environmental testing, as illustrated in Fig. 10. As a key factor to ensuring the link performance, this measurement was particularly important. To measure the peak output power, a linear photodiode was used; the output power was back-calculated from the output current of the photodiode using the circuit and component specifications. The EDFA behaves in an average-power-limited manner, resulting in high instantaneous power. As shown in Fig. 10, the lower duty cycle waveforms (corresponding to higher orders of PPM) produce higher peak output powers. The measured output power tracks closely with the optimal performance, though some efficiency is lost at the highest order of PPM (PPM-128). These measurements, taken under vacuum and in various thermal conditions, serve to validate the functioning of the transmitter on orbit, and confirms the specifications used in previous link budget analyses. In addition to the recorded peak power, the modulated output of the transmitter was examined, as exemplified in Fig. 11. The output of the photodiode shown in this Figure contains a PPM4-modulated message, prepended by a 16-symbol preamble. The steady train of pulses before and after the message provide a consistent duty cycle to maintain the stability of the EDFA.

The CLICK-A PAT system was also thoroughly tested. This involved checking the calibration laser, camera, and FSM actuation, confirming that the beacon detection and standalone calibration procedure could be successfully performed, and measuring pointing performance in vacuum over the operational temperature range of $0-40{ }^{\circ} \mathrm{C}$. The PAT performance was measured using the IR camera in the GSE, which measures the transmit spot and beacon spot locations via a Gaussian fit centroiding algorithm that first fits the larger transmit spot before subtracting it from the image to fit the smaller beacon spot (See Figure 12a). The distance between the two centroids in pixels is measured and converted to angular error using the properties of the IR camera optics: $100 \mathrm{rrad} /$ pixel. The Gaussian statistics for angular pointing error in each axis are computed, and the worst mean 

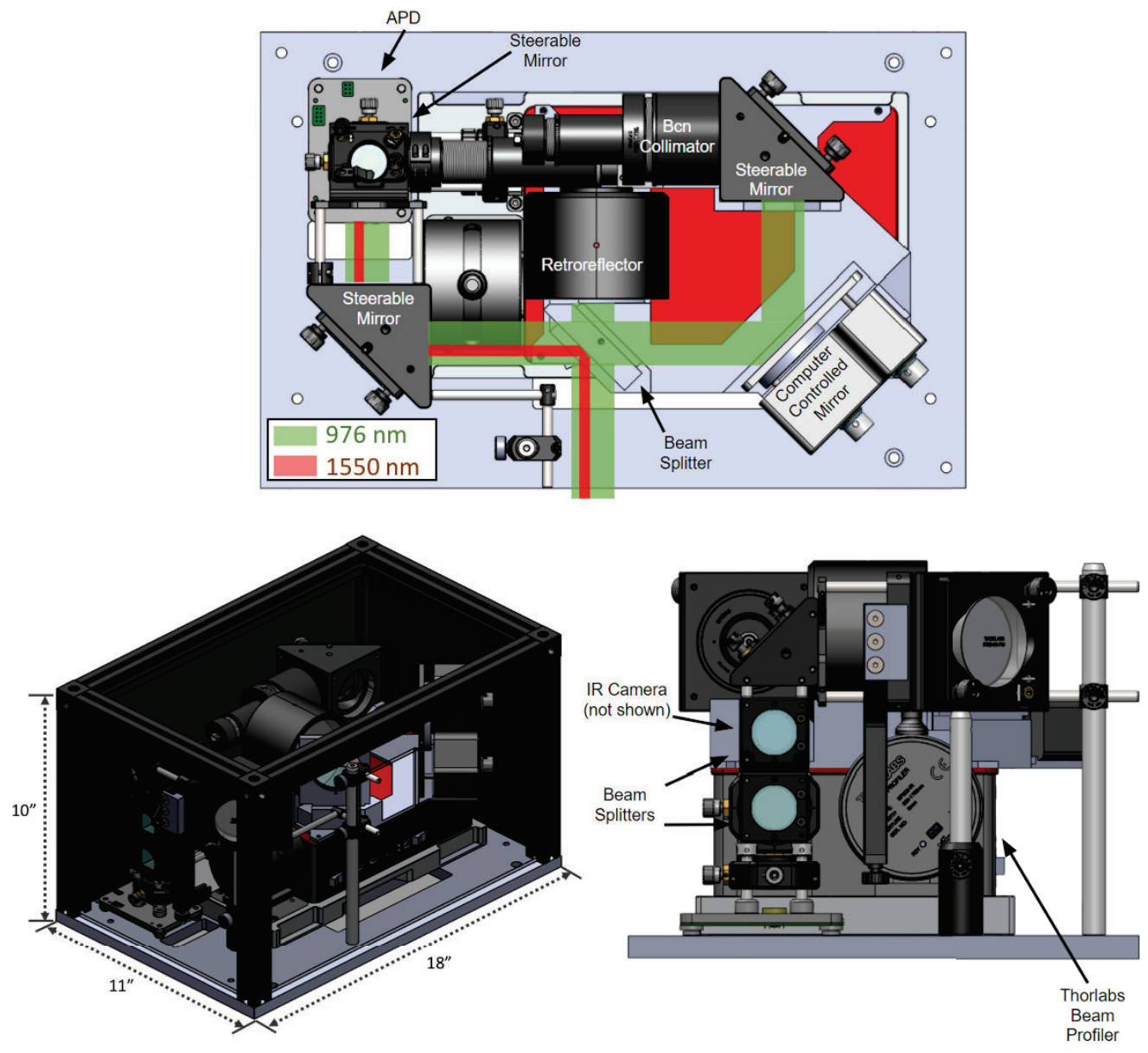

Figure 8: CLICK Payload Testing Ground Support Equipment (GSE)

and standard deviation values from each axis are input to the pointing budget for link budget analysis. The details of the pointing budget and link analysis can be found in Ref. 7. In brief, a simulated typical LEO-ISS overpass of the optical ground station location at MIT Wallace Astrophysical Observatory was used to generate range data (the minimum range is $517.6 \mathrm{~km}$ ). The pointing and link budgets were run over the pass to generate the time-varying performance data. Figure $12 \mathrm{~b}$ shows the link margin performance for the ambient temperature vacuum test. The mission requirement of $\geq 10 \mathrm{Mbps}$ was shown to be satisfied for PPM orders less than or equal to 32 . The performance details are listed in Table 2 .

Table 2: CLICK-A PAT Performance Summary

\begin{tabular}{|c|c|c|}
\hline Test Conditions & Measured Pointing Error Distribution & Estimated PPM-32 Margin at Close Range \\
\hline Cold, $1{ }^{\circ} \mathrm{C}$ & $N(119.2,148.7) \mathrm{\mu rad}$ & $2.8 \mathrm{~dB}$ \\
\hline Ambient, $20^{\circ} \mathrm{C}$ & $N(69.5,20.7) \mathrm{\mu rad}$ & $4.1 \mathrm{~dB}$ \\
\hline Hot, $38^{\circ} \mathrm{C}$ & $N(15.6,106.7) \mathrm{\mu rad}$ & $3.8 \mathrm{~dB}$ \\
\hline
\end{tabular}

It should be noted that the measured pointing error is only one component of the pointing budget, the full details of which are found in Ref. 7. In summary, the PAT performance requirement was met for vacuum tests across the operational temperature range.

In addition to the testing of the CLICK-A payload itself, the interface between the payload and the BCT bus 


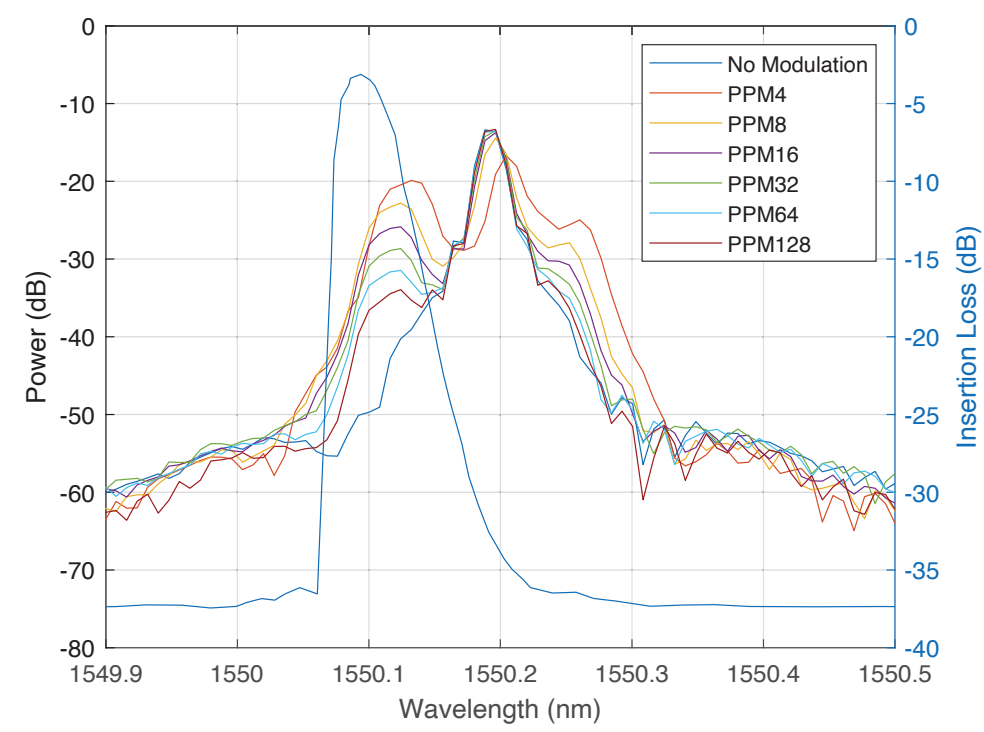

Figure 9: CLICK-A Transmitter Seed Laser Performance

TX APD Test (Vacuum)

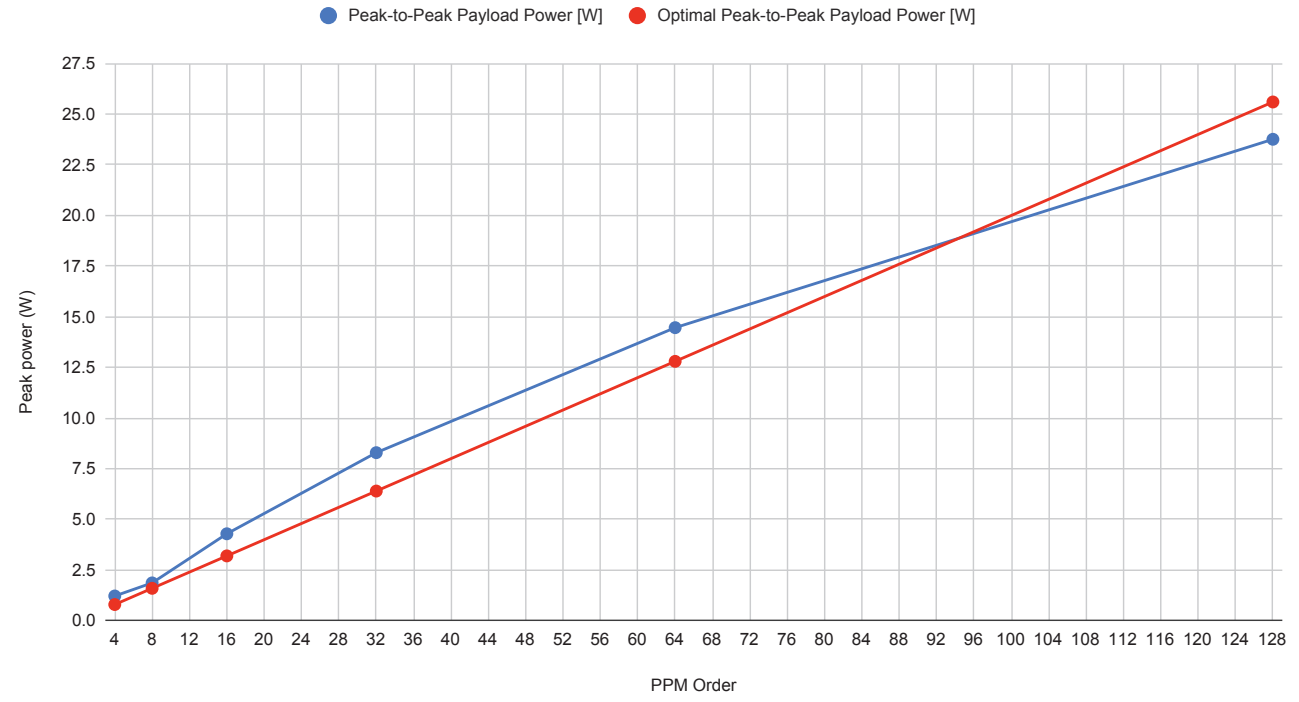

Figure 10: CLICK-A Transmitter Output Peak Power

system was tested. This was enabled by the use of an engineering demonstration unit (EDU) of the bus, which not only provided the bus data interface to the payload but was also capable of routing command and telemetry and interfacing with the MOC computer through the COSMOS mission control software application. This allowed for the parallel development of mission operations scripts and the end-to-end testing of the payload using the COSMOS software, simulating the on-orbit operations scenario. In this manner, all commands, telemetry, and the payload reprogramming capability were tested and confirmed. 


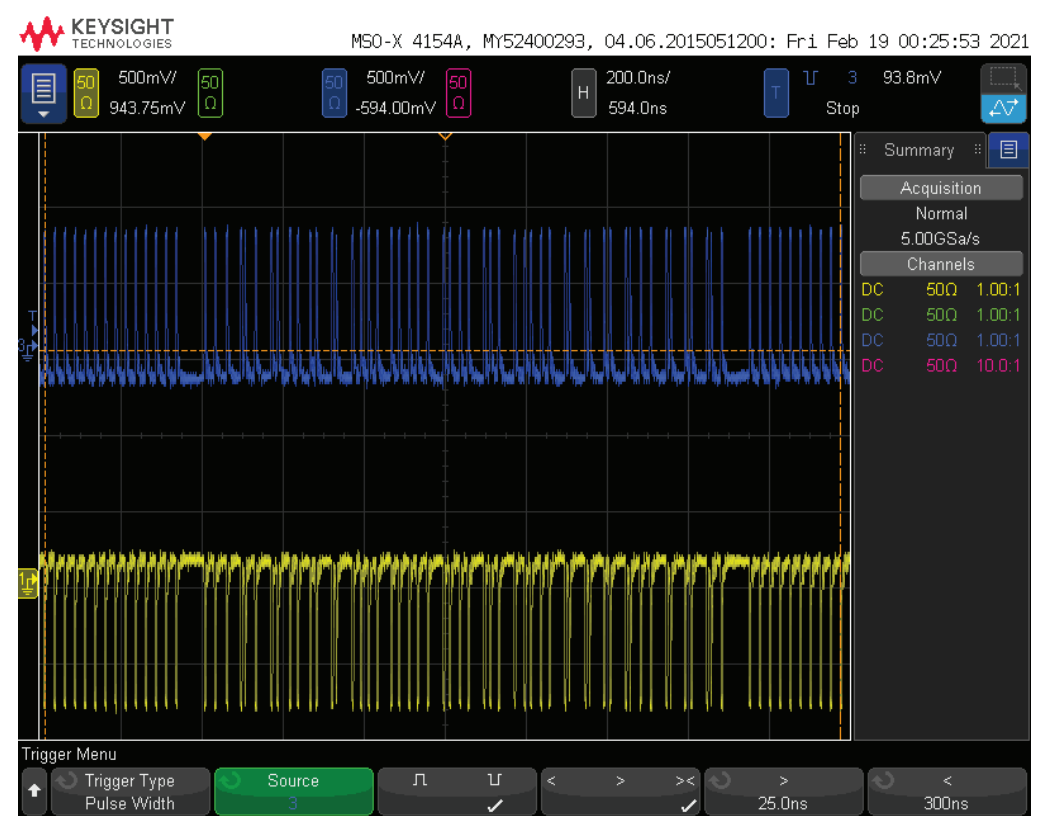

Figure 11: CLICK-A Modulated Output
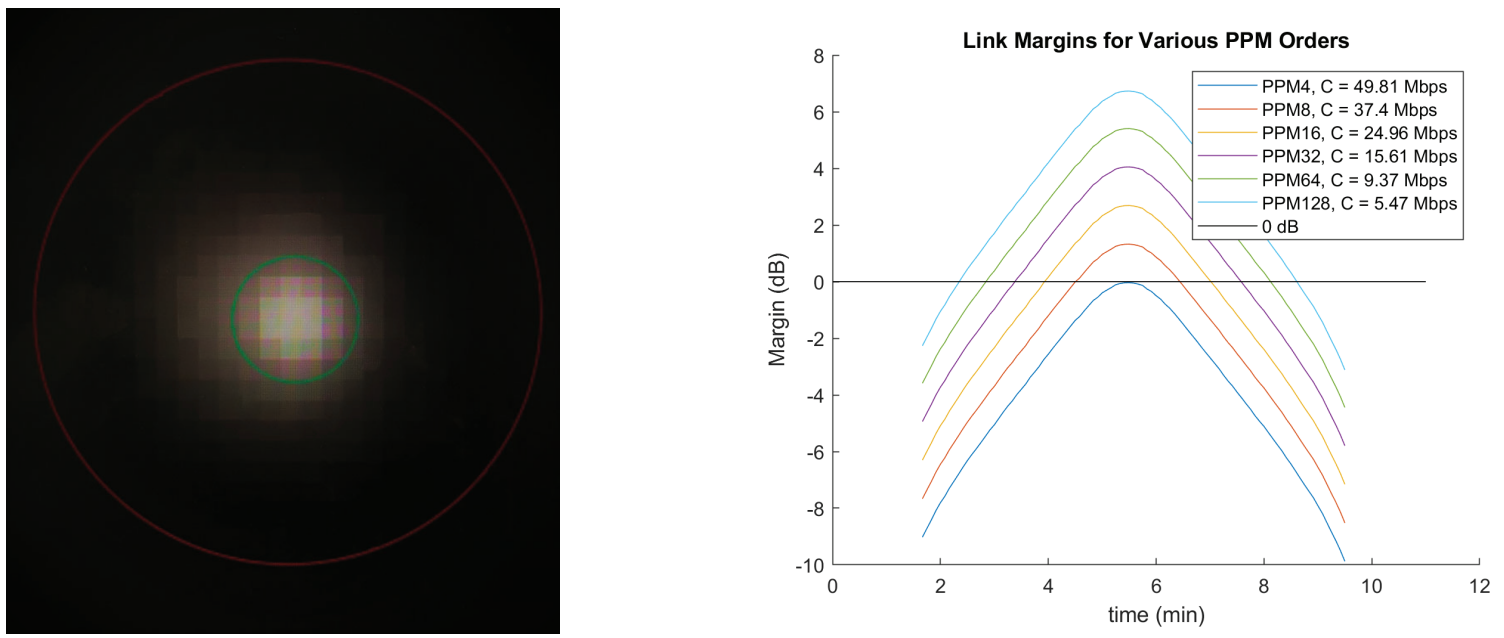

(a) IR camera image of transmit (larger spot outlined in red) (b) Link budget simulation result for ambient temperature and beacon (on top of Tx outlined in green). vacuum PAT test.

Figure 12: CLICK-A PAT Performance During TVAC Testing

\section{CLICK-B/C}

The CLICK-B/C mission consists of two identical CubeSats, launched together to demonstrate full-duplex crosslinks operating with data rates $\geq 20 \mathrm{Mbps}$ over separation distances ranging from $25 \mathrm{~km}$ to $580 \mathrm{~km}$. A ranging capability better than $0.5 \mathrm{~m}$ and time transfer at $200 \mathrm{ps}$ timing accuracy are targeted as well. Similar to CLICK-A, the spacecraft will also be capable of conducting $\geq 10$ Mbps downlinks to the PorTeL OGS.

The design of the CLICK-B/C payload and its mission operations leverage the heritage of the CLICK-A mission. There are also several new elements of the payload system architecture, including the beacon laser and detector system necessary to conduct the crosslink. During the initial CSCTL stage of the crosslink, the beacon lasers will be transmitted by each CubeSat towards the other for alignment and received using the beacon tracking camera. The beacon signal will then be acquired using a quadrant photodiode detector, or quadcell. 
The quadcell is used in the FST mode, for aligning transmit laser using the FSM. Finally, this allows the received transmitter signal to be aligned with the communications receiver, an avalanche photodiode (APD). The signal received by the APD will be processed in real-time for communications or ranging. The full mission ConOps are described in detail in Ref. 9.

Table 3: CLICK-B/C Laser Specifications

\begin{tabular}{|l|l|l|l|l|}
\hline & Wavelength & Average Power & Beam Divergence & Modulation \\
\hline Payload Beacon Laser & $976 \mathrm{~nm}$ & $250 \mathrm{~mW}$ & 22 mrad FWHM & Sine wave \\
\hline Transmitter Laser & $\begin{array}{l}1537 \mathrm{~nm} ; \\
1563 \mathrm{~nm}\end{array}$ & $200 \mathrm{~mW}$ & 71 urad FWHM & M-ary PPM \\
\hline
\end{tabular}

The specifications of the CLICK-B/C laser links are shown in Table 3. Notably, CLICK-B and CLICK-C operate with slightly different wavelengths: $1537 \mathrm{~nm}$ for CLICK-B and $1563 \mathrm{~nm}$ for CLICK-C to enable spectral isolation during duplex operation. The same FSM and beacon tracking camera assembly as CLICK-A are utilized to reduce risk. An overview of the payload structure is shown in Fig. 13 and the optical system is detailed in Fig. 14. In addition to the optomechanical design, significant complexity has been added to the design of the control electronics. For the receiver, both a time-to-digital converter (TDC) as well as pulse recovery and matched filtering are implemented. A detailed description of the receiver design can be found in Ref. 10, and an analysis of the performance of the crosslink and downlink is provided in Ref. 9

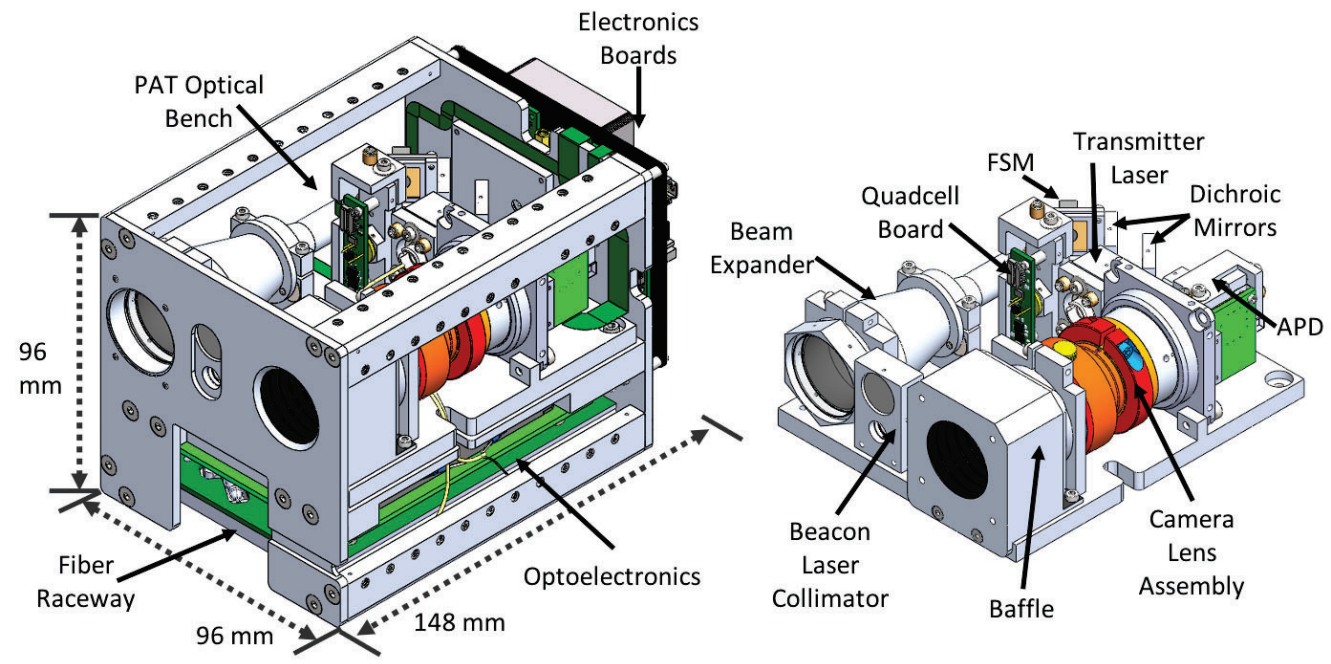

Figure 13: CLICK-B/C Payload Structure

\subsection{CLICK-B/C Status}

The bulk of recent developments has focused on the final steps of the CLICK-A payload. However, concurrent work on CLICK-B/C has allowed this effort to progress as well, primarily on the payload electronics. Currently, a complete set of boards has been fabricated and is under test. Software development has seen the FPGA controlling the payload peripherals to be working. In addition, a new tunable seed laser, capable of emitting any wavelength within $1535 \mathrm{~nm}$ and $1565 \mathrm{~nm}$ has been demonstrated. Using a semiconductor optical amplifier as a modulator, $10 \mathrm{~ns}$ pulses have been achieved. Regarding other elements of the payload, the pointing detector, a quadcell, has been previously tested. ${ }^{10}$ The detection, sub-modulation filtering and FSM control are functional. The APD circuit used in the payload is identical to the PorTeL and OGS APD. The optical bench of the payload has been assembled, and optical characterization of the prototype is ongoing. 


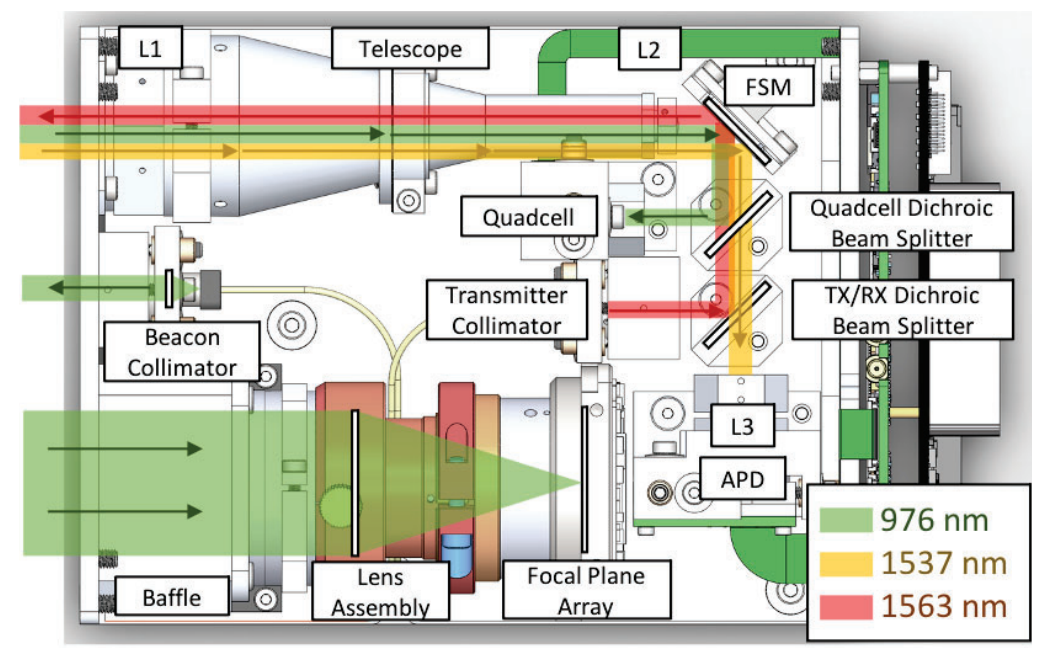

Figure 14: CLICK-B/C Payload Optical Design

\section{CONCLUSION}

This paper presents the updated status on the CLICK mission, including the development and testing of the CLICK-A and CLICK-B/C payloads. For CLICK-A, the final assembly and testing has been completed and the payload has recently been delivered for integration with the spacecraft bus. Prior to delivery, the operation and performance of the transmitter and PAT system were thoroughly tested and characterized. Environmental testing of the payload was conducted and the requirements for on-orbit operation were shown to be met. Future steps include the integration of the payload with the bus system, the completion of the PorTeL OGS, and the development of the ground control and mission operations software in preparation for launch later this year. For CLICK-B/C, the design of the payload electronics has been completed and the boards have been fabricated. The optoelectronics components are currently being demonstrated and tested. The optical bench of the payload has been assembled, the characterization of which is ongoing. The CLICK-B/C development and testing will continue in anticipation of launch in mid-2022.

\section{ACKNOWLEDGMENTS}

The CLICK mission is a collaboration between the MIT Space, Telecommunications, Astronomy, and Radiation (STAR) Lab, University of Florida's Precision Space Systems Lab (PSSL), and NASA Ames Research Center (ARC). This work was funded by the CLICK (CubeSat Laser Infrared CrosslinK) Technology Demonstration Mission, Grant Number 80NSSC18K1579. The views, opinions, and/or findings contained in this work are those of the authors and should not be interpreted as representing the official views or policies, either expressed or implied, of the National Aeronautics and Space Administration.

\section{REFERENCES}

[1] Clements, E., Aniceto, R., Barnes, D., Caplan, D., Clark, J., del Portillo, I., Haughwout, C., Khatsenko, M., Kingsbury, R., Lee, M., Morgan, R., Twichell, J. C., Riesing, K., Yoon, H., Ziegler, C., and Cahoy, K., "Nanosatellite optical downlink experiment: design, simulation, and prototyping," Optical Engineering 55(11), 1 - 18 (2016).

[2] Payne, C., Aguilar, A., , Barnes, D., Diez, R., Kusters, J., Grenfell, P., Aniceto, R., Sackier, C., Allan, G., and Cahoy, K., "Integration and testing of the nanosatellite optical downlink experiment," in [Proceedings of the AIAA/USU Conference on Small Satellites], (2018). https://digitalcommons.usu.edu/cgi/viewcontent.cgi?article=4141\&context=smallsat.

[3] Barnwell, N. S., Parry, S., Ritz, T., Serra, P., and Conklin, J., "Laser amplifier system in a deep space optical transmitter for small satellites," in [AIAA SPACE and Astronautics Forum and Exposition], (2017). 
[4] Conklin, J. W., Nydam, S., Ritz, T., Barnwell, N., Serra, P., Hanson, J., Nguyen, A. N., Priscal, C., Stupl, J., Jaroux, B., and Zufall, A., "Preliminary results from the chomptt laser time-transfer mission," in [Proceedings of the AIAA/USU Conference on Small Satellites], (2019). https://digitalcommons.usu.edu/cgi/viewcontent.cgi?article $=4407 \&$ context=smallsat.

[5] Riesing, K., Yoon, H., and Cahoy, K., "A portable optical ground station for low-earth orbit satellite communications," in [2017 IEEE International Conference on Space Optical Systems and Applications (ICSOS)], 108-114 (2017).

[6] Čierny, O. and Cahoy, K. L., "On-orbit beam pointing calibration for nanosatellite laser communications," Optical Engineering 58(4), $1-8$ (2018).

[7] Čierny, O., Serra, P., Kammerer, W., Grenfell, P., Gunnison, G., Kusters, J., Payne, C., Do Vale Periera, P., Cahoy, K., Ritz, T., Conklin, J., Mayer, D., Stupl, J., and Hanson, J., "Testing of the cubesat laser infrared crosslink (click-a) payload," in [Proceedings of the AIAA/USU Conference on Small Satellites], (2020). https://digitalcommons.usu.edu/cgi/viewcontent.cgi?article $=4617 \&$ context $=$ smallsat.

[8] Kingsbury, R. W., Optical Communications for Small Satellites, PhD thesis, Massachusetts Institute of Technology (9 2015).

[9] Grenfell, P., Serra, P., Čierny, O., Kammerer, W., Gunnison, G., Kusters, J., Payne, C., Cahoy, K., Clark, M., Ritz, T., Coogan, D., Conklin, J., Mayer, D., Stupl, J., and Hanson, J., "Design and prototyping of a nanosatellite laser communications terminal for the cubesat laser infrared crosslink (click) b/c mission," in [Proceedings of the AIAA/USU Conference on Small Satellites], (2020). https://digitalcommons.usu.edu/cgi/viewcontent.cgi?article=4639\&context=smallsat.

[10] Serra, P., Čierny, O., Diez, R., Grenfell, P., Gunnison, G., Kammerer, W., Kusters, J., Payne, C., Murphy, J., Sevigny, T., Do Vale Periera, P., Yenchesky, L., Cahoy, K., Clark, M., Ritz, T., Coogan, D., Conklin, J., Mayer, D., Hanson, J., and Stupl, J., "Optical communications crosslink payload prototype development for the cubesat laser infrared crosslink (click) mission," in [Proceedings of the AIAA/USU Conference on Small Satellites], (2019). https://digitalcommons.usu.edu/cgi/viewcontent.cgi?article=4408\&context=smallsat. 